De la Iglesia-Villasol, M.C. (2019). Huellas de los estudiantes en las plataformas virtuales. Aplicación para evaluar una metodología de aprendizaje activo. Revista Electrónica Interuniversitaria de Formación del Profesorado, 22(3), 173-191.

\title{
Huellas de los estudiantes en las plataformas virtuales. Aplicación para evaluar una metodología de aprendizaje activo.
}

\author{
$M^{a}$ Covadonga de la Iglesia Villasol \\ Universidad Complutense de Madrid
}

\section{Resumen}

La orientación del sistema educativo español hacia escenarios de enseñanza-aprendizaje que traspasan los muros de las aulas físicas hacia plataformas virtuales, abre canales asincrónicos de aprendizaje, y lleva a replantearse cómo aprenden los estudiantes, qué usos hacen de las plataformas virtuales, cómo se interrelacionan con ellas y entre sí, y cómo adquieren conocimientos y desarrollan competencias. El trabajo aborda la implementación de la metodología Aprendizaje Basado en un Proyecto Docente (ABPD) a través del análisis descriptivo de los usos y registros estadísticos, que a modo de huella, dejan los estudiantes en cursos presenciales en el campus virtual, plataforma moddle. Para ello se establecen, según una rúbrica prefijada, unos marcadores que identifican los avances en el proceso de aprendizaje, y se complementa con información de cuestionarios ad-hoc, así como las calificaciones obtenidas y sus correlaciones. A modo de caso se presentan resultados descriptivos de un grupo acumulado de estudiantes del Máster de Formación del Profesorado, en la especialidad de Economía y Administración de Empresas de la UCM, que permiten definir tipologías diversas en su aproximación a los materiales, con patrones diferenciados de aprendizaje según las brechas digitales.

\section{Palabras clave}

Registros de usos; aprendizaje; competencias; learning analytics.

\section{Contacto:}

M Covadonga de la Iglesia Villasol. civ@ccee.ucm.es. Facultad de Ciencias Económicas y Empresariales. Universidad Complutense de Madrid. Campus de Somosaguas. Madrid 28223 Madrid. 


\title{
Footprints of Students on Virtual Platforms. Application to evaluate an Active Learning Methodology
}

\begin{abstract}
The orientation of the Spanish educational system towards teaching-learning scenarios that transcend the walls of physical classrooms towards virtual platforms, opens asynchronous learning channels, and leads to rethinking how students learn, what uses they make of virtual platforms, how they interrelate with them and with each other, and how they acquire knowledge and develop competences. The work addresses the implementation of the Learning Based on a Teaching Project (ABPD) methodology through the descriptive analysis of the uses and statistical registers, which as a footprint, leave the students in face-to-face courses in the virtual campus, moddle platform. For this purpose, markers that identify progress in the learning process are established, according to a prefixed rubric, and supplemented with information from ad-hoc questionnaires, as well as the grades obtained and their correlations. As a case, descriptive results are presented of an accumulated group of students of the Master's Degree in Teacher Training, in the specialty of Economics and Business Administration of the UCM, which allow to define different typologies in their approach to materials, with differentiated patterns of learning according to the digital gaps.
\end{abstract}

\section{Key words}

Records of uses, students, learning, statistics, skills, learning analytics.

\section{Introducción}

Desde la incorporación del Espacio Europeo de Educación Superior (EEES) en el sistema educativo español, se han diseñado múltiples entornos de aprendizaje que coexisten con total naturalidad, con el foco puesto en un aprendizaje centrado en el estudiante, activo, autónomo, crítico y reflexivo (Herrero, 2014), en paralelo a un desarrollo competencial que se presupone ha intensificado (o adquirido) durante su etapa formativa y que es requerido en el entorno profesional, más allá de la necesaria adquisición de contenidos específicos.

Este trabajo recoge el análisis descriptivo de la explotación de las huellas, registros o rastros que los estudiantes dejan en las plataformas digitales, en interacción con la información institucional, calificaciones y valoraciones del proceso de aprendizaje, en un curso en el que se ha implementado la metodología de trabajo activa y radial, Aprendizaje Basado en un Proyecto Docente (en adelante ABPD, según Iglesia, 2018), que adapta a la formación de futuros docentes los principios del aprendizaje basado en problemas (Boss y Krauss, 2007; Bender, 2012 y Patton, 2012). La ficha metodológica de ésta estrategia se muestra en la figura 1 del Anexo, y se sustenta en el binomio creatividad-innovación, cuya esencia es contribuir a la mejora de la significatividad del aprendizaje de los estudiantes, utilizando el aula virtual y otras plataformas en abierto como un complemento asincrónico necesario a la enseñanza presencial. Dicha propuesta metodológica, válida en la era digital, se aplica en el bloque temático de Didáctica de Economía y Administración, en el Máster de Formación del Profesorado, en la UCM, y simula los pasos, procesos y documentos del trabajo profesional que realizarán los estudiantes posteriormente cuando se incorporen al desempeño profesional. El contenido temático que trabajan los estudiantes en la metodología ABPD se articula en torno a una programación integral, que a forma de árbol se abre en unidades 
docentes, para las que elaboran sus propios materiales, recursos y propuestas docentes, pasando por las fases de autoevaluación y evaluación por pares, y siempre alineados con las competencias del currículum en la ESO y el Bachillerato1 que actúa de marco normativo de referencia.

Las actividades diseñadas para ese aprendizaje activo segmentan al grupo en subgrupos, formados por estudiantes con personalidades, tipos de inteligencias, formas y ritmos de aprendizaje diversos, por lo que es necesario para el docente prefijar marcadores secuenciales del desarrollo y aprendizaje autónomo (Cano, 2009). Es decir, el docente observa, tanto en las tareas individuales como colectivas, el progreso del estudiante en la adquisición de conocimientos, y los usos y huellas que su interrelación con los entornos digitales va dejando.

Ese rastro dejado en las plataformas digitales, es un extraordinario banco de datos que está siendo objeto de explotación, generando una creciente y fructífera literatura empírica, Learning Analytics (en adelante LA, analítica del aprendizaje), y que puede facilitar que el docente conozca cómo asincrónicamente trabajan los estudiantes, ritmos, frecuencias, franjas horarias, repetición de accesos, tiempos de usos, etc. Además, en su caso, desde el feed-back de su conocimiento, el docente puede reelaborar una estrategia de aprendizaje diferenciada si anticipa el análisis de la información que el estudiante va dejando. La definición oficial del término Learning Analytics fue dada en la convocatoria de la First International Conference on Learning Analytics and Knowledge (LAK 2011) y adoptada por la reciente Society for Learning Analytics Research (SoLAR), como la medición, recopilación, análisis e informe de datos sobre los alumnos y sus contextos, con el fin de comprender y optimizar el aprendizaje y los entornos en los que se produce. En ésta línea de investigación se inserta el trabajo, que aporta un análisis descriptivo de la explotación de los registros que los estudiantes dejan en el uso de las herramientas digitales, en interacción con la información institucional, calificaciones y valoraciones del proceso de aprendizaje, en un curso en el que se ha implementado la metodología ABPD.

\section{Algunos elementos del estado de la cuestión.}

Dos aspectos de la literatura concentran el interés para contextualizar el trabajo, que inciden en la evaluación de la estrategia activa ABPD que realizan los estudiantes, como son los roles actuales del docente y la masiva información registrada a partir de los usos que los estudiantes realizan en las plataformas de aprendizaje digitales, sin que sea una revisión exhaustiva, hecho que excedería los objetivos de este trabajo empírico.

El docente, sea de la etapa formativa que sea, asume nuevos roles en el ejercicio de su profesión, como diseñador de nuevos entornos de aprendizaje, generador y evaluador de recursos útiles para el autoaprendizaje, orientador, tutor, motivador, en un continuo aprendizaje de su profesión, encaminado tanto a transmitir conocimiento técnico, especializado como a favorecer el desarrollo de competencias requeridas en el entorno profesional (Iglesia, 2006). Harrison y Killion (2007) asumen que será un facilitador del éxito en el aprendizaje de los estudiantes, especialista curricular, un proveedor de recursos, instructor, facilitador de apoyo en el aula y del aprendizaje, mentor de sus estudiantes, analista de datos y catalizador del cambio. Para Viñals y Cuenca (2016), los nuevos roles pasan por convertirse en un organizador, guía, generador, acompañante, coacher, gestor de aprendizaje, orientador, facilitador, tutor, dinamizador o asesor, y que cambian la

\footnotetext{
${ }^{1}$ Real Decreto 1105/2014, de 26 de diciembre, establece el currículo básico de la Educación Secundaria Obligatoria y del Bachillerato, siendo las competencias: Comunicación lingüística; Competencia matemática y competencias básicas en ciencia y tecnología; Competencia digital; Aprender a aprender; Competencias sociales y cívicas; Sentido de iniciativa y espíritu emprendedor; Conciencia y expresiones culturales.
} 
transmisión unidireccional del conocimiento por el intercambio horizontal de información. Más aún, Prensky (2013) agrupa los roles del profesor competente digital2 en tres epígrafes para conseguir un efectivo, motivador y atractivo aprendizaje: ser entrenador (observación individual y motivación al estudiante), ser guía (ayudar a conseguir objetivos) y ser experto en la instrucción (a través del conocimiento y la creatividad), quien utiliza la tecnología disponible para mejorar la metodología del proceso de enseñanza-aprendizaje (Bates, 2015).

Siendo así, el docente de futuros profesores (en formación) asume, además, el rol de observador del aprendizaje de los estudiantes, en su desarrollo de competencias y simulación de la profesión docente, para lo cual la gestión y el análisis de la información (analista de datos a que se refiere Harrison y Killion, 2007) le permite conocer mejor su propia acción docente, la tipología de sus estudiantes y los resultados, actitudes y compromiso con el programa.

Long y Siemens (2011) afirman con rotundidad que el análisis de los datos extraídos de la acción docente tiene un papel significativo en el futuro de la Educación Superior para penetrar en la niebla instalada, tanto guiando a futuras reformas en las actividades y programas como mejorando la acción docente durante el ciclo completo del estudiante: curso y nivel, minería de datos educativos, currículum inteligente, contenido y aprendizaje adaptativo. Siemens y Baker (2012) abogan por una comunicación y colaboración mayor y formal entre las diversas comunidades de investigación, Minería de datos educativos (EDM), Learning Analytics (LA) and Knowledge (LAK), que, si bien muestran diferencias en los descubrimientos, las reducciones, los orígenes, la adaptación y personalización, o las técnicas, son salvables hasta compartir investigaciones, métodos y herramientas para la extracción y el análisis de datos. Así, el Learning Analytics toma un papel significativo tanto para i) los profesores/educadores, para conocer a tiempo real el comportamiento y rendimiento de los estudiantes e identificar si están en riesgo de abandonar el curso o necesitar ayuda para recuperar el ritmo del aprendizaje; ii) los estudiantes que pueden recibir información particularizada de su progreso, como elemento motivador; y iii) los responsables para conocer, por ejemplo, el uso eficiente de los recursos, las ventajas competitivas, las mejoras en la calidad y rendimiento de la experiencia de aprendizaje. Autores como Sampson (2017) visualizan un futuro aprendizaje personalizado, aplicando la analítica del aprendizaje en análisis descriptivos, predictivos y/o prescriptivos de recomendaciones, resultado de parametrizar la información para definir perfiles, a partir de datos estáticos (aspectos demográficos, sociales, familiares, o del pasado académico) y datos dinámicos (referidos al proceso de aprendizaje: compromiso, comportamiento, y rendimiento).

Romero y Ventura (2007) consideran la aplicación de la minería de datos en sistemas de elearning en su ciclo iterativo, que guía, facilita y mejora el aprendizaje en su conjunto, convirtiendo los datos en conocimiento y filtrándolo para la toma de decisiones, identificando el proceso de extracción de datos (recoger datos, pre-procesar, aplicar algoritmos, interpretar, evaluar y desplegar resultados), siendo ésta una referencia imprescindible sobre las aplicaciones del uso del registro de datos, por la extensa sistematización y clasificación que realizan por el tipo de datos, técnicas utilizadas o tareas educativas que resuelven. Ferguson (2012) señala los factores que impulsan el desarrollo exponencial del LA: i) generación de grandes matrices de datos (big data) para extraer valor de los datos relacionados con el aprendizaje; ii) el aprendizaje en línea (online learning), que constituye el reto de optimizar las oportunidades del aprendizaje en línea, y iii) intereses

\footnotetext{
2 El informe A Framework for Developing and Understanding Digital Competence in Europe (2013, Ferrari, A.), indica que se es competente digital si se cubren las dimensiones referidas a la búsqueda y gestión de la Información digital; comunicar e interactuar; creación de contenido multiformato y multimedia; identificar y resolver problemas, y protección y seguridad digital de la información. http://publications.jrc.ec.europa.eu/repository/bitstream/JRC83167/lb-na-26035-enn.pdf
} 
políticos (political concerns) y económicos, para optimizar los resultados educativos y de aprendizaje, a nivel nacional o internacional. Estos retos llaman la atención sobre tres grupos de agentes diferentes, gobiernos, instituciones educativas y profesores, cuyos intereses se superponen y abordan el trabajo analítico a diferentes escalas y profundidad.

El alcance del LA está siendo masivamente incorporado por Universidades y Centros Educativos, tanto para evaluar planes formativos formales como informales (Ferguson, Brasher, Clow y otros, 2016), inmersos en el Plan Opening up Education (Comisión Europea, 2013) para la promoción e investigación en la adaptación de tecnologías y juegos digitales de aprendizaje y su posterior analítica, diseñados desde disciplinas como la estadística, la inteligencia artificial, la ciencia computacional, las ciencias de la educación, o desde ámbitos de diseño de perfiles psicológicos y de aprendizaje (Siemens, Dawson y otros, 2013). En las manos de los docentes e investigadores está ir ampliando las fronteras que delimitan las aplicaciones de LA a la docencia e investigación.

\section{Objetivo}

El objetivo de esta investigación es analizar y evaluar diversos aspectos de la implementación de la estrategia de aprendizaje integradora y activa ABPD, desarrollada como un caso de estudio. Este objetivo se concreta en otro más específico, que es valorar la utilidad de la estrategia a partir de la interacción de la información extraída de los usos digitales que hacen los estudiantes, con las calificaciones obtenidas y las valoraciones o grado de satisfacción que muestran con la misma.

Dada la particularidad del curso y la tipología de estudiantes, quienes cursan el MFP en la especialidad ECO y ADE (UCM) para convertirse en futuros docentes, la estrategia de aprendizaje ABPD integra y reproduce fases y procesos de la acción docente, y es observada por el docente desde la extracción y el análisis de la información que se genera, en un proceso de continuo reajuste metodológico.

\section{Metodología del estudio de caso}

Desde la explosión del LA en 2011, la evaluación de las herramientas utilizadas en el aprendizaje es otro aspecto relevante en la literatura empírica, en general sustentada por el uso que hacen de ellas las instituciones o colectivos de investigadores. Para este trabajo se optó por seguir un diseño cuantitativo, de tipo descriptivo no experimental, que combina datos de los registros de usos y huellas que los estudiantes dejan en el aula virtual, combinados con cuestionarios con las valoraciones que los estudiantes realizan de las diversas etapas que se siguen en el programa formativo, y las calificaciones que obtienen en las diversas fases del programa formativo.

\section{Población participante}

A modo de ficha técnica, la tabla 1 describe los cursos y el número de estudiantes que voluntariamente han participado en el estudio de caso que se presenta, de seguimiento y registro (SEG). Si bien el campus virtual por defecto facilita las huellas de todos los matriculados, no todos consintieron la observación de su huella o rastro en el aprendizaje y el cruce con informaciones de otros registros, como son los breves cuestionarios sobre aspectos personales y profesionales, atributos docentes y de satisfacción con la metodología. Dicho consentimiento requería, obviamente, seguir unas normas, 
disponibilidad, dedicación exigible y roles a los que los estudiantes debían comprometerse3. Los resultados se presentan únicamente para tres cursos académicos4, para los cuales la metodología ABPD estaba consolidada, fijándose el rango mínimo de participación de los estudiantes en el seguimiento en el $65 \%$, superándose en todos los cursos.

Tabla 1.

Distribución de estudiantes en el programa de seguimiento (SEG) del ABPD

\begin{tabular}{lccc}
\hline Cursos & Total SEG ( $>65 \%$ total curso) & Género (\% varones) & $<30$ años $(\%)$ \\
\hline $\mathbf{2 0 1 4 - 1 5}$ & 19 & $47.37 \%$ & $11(57.89 \%)$ \\
\hline $\mathbf{2 0 1 5 - 1 6}$ & 10 & $40.00 \%$ & $6(60,00 \%)$ \\
\hline $\mathbf{2 0 1 6 - 1 7}$ & 12 & $41.66 \%$ & $6(50,00 \%)$ \\
\hline Total & 41 & $43.90 \%$ & $56.10 \%$ \\
\hline
\end{tabular}

El $43,9 \%$ de los que estudiantes que participan en el seguimiento son varones, y el rango de edades subdividido en tres intervalos muestra que el $56,10 \%$ está entre 24 y 30 años, el $26,83 \%$ entre 30 y 40 años y el resto $(17,07 \%)$ tiene 40 años o más, hecho que describe tres tipologías de estudiantes (no necesariamente en correspondencia con tales intervalos de edad):

i) recientemente graduados, con mínima y precaria experiencia profesional: $26,83 \%$;

ii) con amplia experiencia profesional que voluntariamente o por reajustes laborales, se encuentran en situación de desempleo y deciden reorientar su carrera profesional (39,02\%),

iii) quienes con experiencia profesional mantienen su empleo y declaran que el máster es un complemento formativo para reforzar su currículum (34,15\%).

No es factible realizar un análisis significativo de diferencias entre los diversos subgrupos, por edad, experiencia y sexo, dada la reducida adscripción de estudiantes a los mismos, si bien la percepción descriptiva revela un distinto grado de madurez, experiencial y laboral.

\section{Instrumentos y registros de datos}

Para el análisis descriptivo se han recuperado diversos instrumentos de información primaria, en la línea de lo expuesto por Sampson (2017):

i) Los registros de los usos de las herramientas del campus virtual Moodle y del blog de la asignatura. La referencia para la extracción, la descripción y el análisis de los datos es VelaPérez y otros (2017). En la literatura empírica de los último 10 años se referencian una gran cantidad de herramientas de procesamiento ${ }^{5}$, siendo una referencia imprescindible Romero, Ventura y García (2008).

ii) Las calificaciones según la rúbrica de evaluación de los contenidos a elaborar en el desarrollo del programa de aprendizaje, algunos individuales y otros en grupo, definida ad-hoc, con ítems que recogen niveles de competencia en los recursos que elaboran y presentan los estudiantes, las habilidades expositivas, el aporte creativo, los aspectos didácticos, y la interacción con el grupo. Dicha rúbrica refleja la triple hélice creatividad-digitalizaciónoportunidad, sobre la que pivota la estrategia ABPD que da soporte a este estudio, con el

\footnotetext{
${ }^{3}$ No todos los cursos participa el mismo porcentaje de alumnos en este seguimiento, si bien todos los estudiantes siguen la misma estrategia de aprendizaje, ABPD. Por ejemplo, de los 18 estudiantes del curso 2016_17, sólo participan voluntariamente en el programa de rastreo 12 , el $67 \%$.

${ }^{4}$ Los registros existentes, no están sistematizados para el curso 2017-18.

${ }^{5}$ Tanto generales como específicas, que van desde programas estandarizados DBMiner, SPSS DB2 Intelligent Miner, a numerosas aplicaciones de uso público desarrolladas por colectivos de investigadores.
} 
objetivo de que los recursos generados por los estudiantes sean válidos para el aprendizaje, y versátiles en el contexto en el que los estudiantes desarrollarán su actividad profesional futura.

iii) Los datos de los cuestionarios, algunos ex ante y otros ex post, con los que los estudiantes evalúan tanto la estrategia de aprendizaje desarrollada en el curso, ABPD, como la iniciativa de seguimiento de sus registros si son estudiantes que han participado en la misma.

Respecto a la rúbrica de evaluación, figura 1, incluye lo referido a las actividades y recursos que forman parte de la evaluación continua en el apartado de recursos (epígrafes 1 a 4, definida a partir de Villalustre y del Moral, 2014), a la que se le ha añadido los ítems alusivos a la evaluación que el profesor y el propio estudiante hace de las aportaciones en el foro y/o blog, en la interacción del grupo (epígrafe 5), cuyo desglose se muestra en la figura 2.

\begin{tabular}{|c|c|c|c|}
\hline & \multicolumn{3}{|c|}{ Nivel de competencia } \\
\hline & Bajo & Medio & Alto \\
\hline $\begin{array}{l}\text { 1_USOS } \\
\text { DIGITALES }\end{array}$ & $\begin{array}{l}\text { Escaso uso multiformato. } \\
\text { Gestión baja de componente } \\
\text { formativo }\end{array}$ & $\begin{array}{l}\text { Adecuada utilización de } \\
\text { formatos digitales. } \\
\text { Uso de diversos soportes. }\end{array}$ & $\begin{array}{l}\text { Elevado uso herramientas } \\
\text { digitales diversas. } \\
\text { Buena selección y adecuación a } \\
\text { contenidos. }\end{array}$ \\
\hline $\begin{array}{l}\text { 2_HABILIDADES } \\
\text { EXPOSITIVAS }\end{array}$ & $\begin{array}{l}\text { Poco adecuada o fluida. } \\
\text { Escasa secuencia expositiva. } \\
\text { Incoherencias en la } \\
\text { presentación. }\end{array}$ & $\begin{array}{l}\text { Adecuada temática. } \\
\text { Adecuada secuencia } \\
\text { expositiva. } \\
\text { Adecuada presentación. }\end{array}$ & $\begin{array}{l}\text { Temática claramente definida. } \\
\text { Buena secuencia expositiva. } \\
\text { Coherencia entre contenido y } \\
\text { presentación. }\end{array}$ \\
\hline $\begin{array}{l}\text { 3_APORTE } \\
\text { CREATIVO }\end{array}$ & $\begin{array}{l}\text { Escasa creatividad en los } \\
\text { recursos digitales. }\end{array}$ & $\begin{array}{l}\text { Uso de diferentes recursos } \\
\text { digitales. } \\
\text { Capacidad expresiva limitada. }\end{array}$ & $\begin{array}{l}\text { Potencia la capacidad expositiva. } \\
\text { Originalidad e innovación de los } \\
\text { recursos. }\end{array}$ \\
\hline $\begin{array}{l}\text { 4_ASPECTOS } \\
\text { DIDÁCTICOS }\end{array}$ & $\begin{array}{l}\text { Escaso potencial didáctico. } \\
\text { Confusión en la transmisión } \\
\text { de conocimientos. }\end{array}$ & $\begin{array}{l}\text { Exposición adecuada y } \\
\text { motivadora. } \\
\text { La estrategia didáctica puede } \\
\text { ser mejorable. }\end{array}$ & $\begin{array}{l}\text { Potencia la reflexión y } \\
\text { adquisición de valores. } \\
\text { Uso de estrategias adecuadas. }\end{array}$ \\
\hline $\begin{array}{l}\text { 5_INTERACCION } \\
\text { DEL GRUPO } \\
\text { (VER CUADRO 2) }\end{array}$ & $\begin{array}{l}\text { Escasa interacción. } \\
\text { Baja aportación en las } \\
\text { entradas y documentos }\end{array}$ & $\begin{array}{l}\text { Adecuada interacción con el } \\
\text { grupo. } \\
\text { Entradas y documentos } \\
\text { bastante significativos. }\end{array}$ & $\begin{array}{l}\text { Elevada participación e } \\
\text { interacción. } \\
\text { Entradas con contenido } \\
\text { significativo y concluyente }\end{array}$ \\
\hline
\end{tabular}

Figura 1. Distribución de Rúbrica de Evaluación de recursos didácticos.

\begin{tabular}{|l|l|l|l|}
\hline & \multicolumn{3}{|c|}{ Rúbrica Interacción del Grupo } \\
\hline & \multicolumn{1}{|c|}{ plazos frecuencia } & \multicolumn{1}{c|}{ autoría } & \multicolumn{1}{c|}{ aportaciones extras } \\
\hline $\begin{array}{l}\text { ASPECTOS } \\
\text { FORMALES }\end{array}$ & $\begin{array}{l}\text { Cumplimiento plazos. } \\
\text { Intervenciones regulares. }\end{array}$ & $\begin{array}{l}\text { Autoría: copia, plagio. } \\
\text { Aspectos ortográficos, } \\
\text { redacción. }\end{array}$ & $\begin{array}{l}\text { Documentación adicional: recursos, } \\
\text { enlaces, artículos, ejemplos. } \\
\text { Referencias bibliográficas y citas. }\end{array}$ \\
\hline $\begin{array}{l}\text { ASPECTOS } \\
\text { CONCRETOS }\end{array}$ & $\begin{array}{l}\text { cumplimiento } \\
\text { Rlantean nuevas ideas o líneas. } \\
\text { Argumentan las aportaciones. }\end{array}$ & $\begin{array}{l}\text { Calidad de los materiales. } \\
\text { Relación con el tema de trabajo. } \\
\text { Originalidad de exposiciones. }\end{array}$ & $\begin{array}{l}\text { Aportan feed-back a compañeros. } \\
\text { Evalúan significativamente. } \\
\text { Integran las diversas opiniones. }\end{array}$ \\
\hline
\end{tabular}

Figura 2. Rúbrica específica de la integración del grupo

La información personal de los estudiantes ha sido gestionada bajo el uso de un algoritmo de identificación anónima6. La existencia de numerosas herramientas de gestión de datos, con un alcance cada vez mayor, ha planteado entre diversos colectivos la preocupación sobre cuestiones de privacidad, aspectos éstos muy pertinentes, abordados inicialmente por Campbell (2007), quién fija un marco basado en definiciones, valores, principios y lealtades.

\footnotetext{
${ }^{6}$ Conciliar la investigación con las nuevas normas europeas de protección de datos (Europe's General Data Protection Regulation, GDPR, de 2016) es necesario y está generando cambios en el uso futuro del LA, por cuanto las instituciones tienen responsabilidad y obligaciones en la protección.
} 


\section{Resultados del estudio de caso}

Se presentan resultados descriptivos recogidos en la implementación de la estrategia de aprendizaje $A B P D$, algunos genéricos para el trienio de análisis y otros particularizados para el curso 2016-17, tanto de las huellas que dejan los estudiantes por el uso digital, como de las calificaciones de los diversos elementos evaluables durante el curso y las valoraciones que los estudiantes realizan de dicha estrategia y del proceso de registro y seguimiento.

\section{Resultados del registro de usos en plataformas virtuales.}

Para el trienio, tabla 2, se dispone de 12738 registros válidos, correspondiendo el 55,50\% de los registros al curso 2016-17, cuando la estrategia ABPD estaba implementada satisfactoriamente. Una visión rápida del porcentaje de registros por estudiantes y media semanal, muestra la elevada dispersión en los usos de las plataformas digitales. Los estudiantes más activos registran, en media, porcentajes entre el 9,28 y el 9,27\%, mientras que los menos activos están entre el 0,06\% y el 0,08\% del total. Estas medias semanales y sus diferenciales determinan el cálculo de un indicador de brecha de uso digital, computada con respecto al estudiante más activo del trienio analizado, que supera el $99 \%$ respecto de aquellos estudiantes que marcan la cota inferior de uso.

Tabla 2.

Estadísticos total registros: Trienio 2014-15 a 16-17.

\begin{tabular}{|c|c|c|c|c|}
\hline 10 mayores & Registros & \% Total & Media semanal & Brecha uso semanal \\
\hline Curso 2014-15 & 1193 & 9,97 & 66,28 & 0,00 \\
\hline Curso 2015-16 & 1180 & 9,86 & 65,56 & 1,09 \\
\hline \multirow[t]{9}{*}{ Curso 2016-17 } & 1111 & 9,28 & 61,72 & 6,87 \\
\hline & 1051 & 8,78 & 58,39 & 11,90 \\
\hline & 748 & 6,25 & 41,56 & 37,30 \\
\hline & 696 & 5,82 & 38,67 & 41,66 \\
\hline & 461 & 3,85 & 25,61 & 61,36 \\
\hline & 460 & 3,84 & 25,56 & 61,44 \\
\hline & 455 & 3,80 & 25,28 & 61,86 \\
\hline & 410 & 3,43 & 22,78 & 65,63 \\
\hline & Registros & $\%$ Total & Media semanal & Brecha uso semanal \\
\hline \multirow[t]{6}{*}{10 menores } & 24 & 0,20 & 1,33 & 97,99 \\
\hline & 23 & 0,19 & 1,28 & 98,07 \\
\hline & 23 & 0,19 & 1,28 & 98,07 \\
\hline & 21 & 0,18 & 1,17 & 98,24 \\
\hline & 20 & 0,17 & 1,11 & 98,32 \\
\hline & 19 & 0,16 & 1,06 & 98,41 \\
\hline \multirow[t]{2}{*}{ Curso 2016-17 } & 15 & 0,13 & 0,83 & 98,74 \\
\hline & 10 & 0,08 & 0,56 & 99,16 \\
\hline Curso 2015-16 & 8 & 0,07 & 0,44 & 99,33 \\
\hline Curso 2014-15 & 7 & 0,06 & 0,39 & 99,41 \\
\hline Todos, Media & & 100 & 664,94 & \\
\hline
\end{tabular}

Particularizando para el curso 2016-17, tabla 3, se muestran los estadísticos de los 18 estudiantes, ordenados y codificados por orden alfabético7, de los cuales sólo 12 participan

\footnotetext{
${ }^{7}$ Estadísticamente, la correlación (Pearson) entre el coeficiente de correlación entre la ordenación según orden alfabético de los alumnos y sus registros de uso es 0,0885 , aspecto meramente aleatorio.
} 
en el programa de seguimiento (SEG), con muy diversa frecuencia, presencia y actividad digital. De las 18 semanas de seguimiento realizado en el trimestre en el que se imparte el bloque temático de Didáctica, se registran NR=6535 usos válidos entre los estudiantes que participan en el SEG, que representan el 92,43\% del total del curso $(\mathrm{NT}=7070)$, con una media semanal de 363,26 registros y una media por estudiante y semana de 30,25 (siendo para el total de estudiantes del curso, 392,78 registros por semana y 21,82 por estudiante y semana). Para quienes no consintieron ser parte del plan de seguimiento, la media semanal de registros es de 29,72, mientras que la media por alumno y semana es únicamente del 4,95. El intervalo máximo y mínimo de registros semanal por alumnos va de 61,72 (alumno A6_R) a sólo 0,83 (alumno A16).

Los estudiantes que participan en el SEG presentan, obviamente, porcentajes de uso y medias semanales superiores. En el curso 2016-17, los cuatro estudiantes más activos presentan medias semanales entre 61,72 y 38,67, y los cuatro menos activos solo registran entre 0,83 y 3,67 usos semanales (tabla 3). Estas medias semanales y sus diferenciales determinan la brecha de uso digital, referenciada al alumno más activo, A6_R, y definir una clasificación y tipología básica de estudiantes:

i) proactivos: brecha digital < 25\%: sólo estarían el estudiante de referencia A6_R y A8_R;

ii) activos: brecha entre 25 y el 50\%, incluiría a los estudiantes $A 5 \_R$ y $A 14 \_R$;

iii) inactivos: brecha entre 50 y $75 \%$, incluiría: A5_R, A10_R, A2_R, A9_R, A3_R, A11_R, y A13_R. iv) descolgados: brecha entre el 75 y 100\%: incluiría al resto: $A 12$ _R, A7, A4, A1, A18, A17 y A16.

Tabla 3.

Estadísticos total registros de usos. Curso 2016-17

\begin{tabular}{|c|c|c|c|c|c|c|c|}
\hline & \multicolumn{5}{|c|}{ Todos los alumnos (TOT) NT=7070 } & \multicolumn{2}{|c|}{ Solo SEGUIMIENTO (SEG) } \\
\hline & $\mathrm{N}$ & $\%$ & Media & Dif media & Brecha uso & $\%$ sobre NR & Dif media \\
\hline $\mathrm{A} 1$ & 66 & 0,93 & 3.67 & $-18,15$ & 94.05 & & \\
\hline$A 2 R$ & 410 & 5,80 & 22,78 & 0,96 & 63,09 & 6,27 & $-7,47$ \\
\hline$A 3 R$ & 344 & 4,87 & 19,11 & $-2,71$ & 69,04 & 5,26 & $-11,14$ \\
\hline A4 & 193 & 2.73 & 10.72 & $-11,10$ & 82.63 & & \\
\hline A5 $R$ & 748 & 10,58 & 41,56 & 19,74 & 32,66 & 11,45 & 11,31 \\
\hline A6 R & 1111 & 15.71 & 61.72 & 39.90 & 0,00 & 17.00 & 31,47 \\
\hline A7 & 201 & 2,84 & 11,17 & $-10,65$ & 81,90 & & \\
\hline$A 8 R$ & 1070 & 15,13 & 59.44 & 37.62 & 3.69 & 16.37 & 29.19 \\
\hline A9 $R$ & 408 & 5,77 & 22,67 & 0,85 & 63,27 & 6,24 & -7.58 \\
\hline A10 R & 455 & 6,44 & 25,28 & 3,46 & 59,04 & 6,96 & -4.97 \\
\hline A11 R & 324 & 4.57 & 17,94 & $-3,88$ & 70,93 & 4.94 & $-12,31$ \\
\hline A12 R & 219 & 3,10 & 12,17 & $-9,65$ & 80,28 & 3.35 & $-18,08$ \\
\hline $\mathrm{A} 13 \mathrm{R}$ & 290 & 4.10 & 16.11 & -5.71 & 73.90 & 4.44 & -14.14 \\
\hline A14 R & 696 & 9.84 & 38,67 & 16.85 & 37.35 & 10.65 & 8.42 \\
\hline A15 R & 461 & 6.52 & 25.61 & 3.79 & 58.51 & 7.05 & -4.64 \\
\hline $\mathrm{A} 16$ & 15 & 0,21 & 0.83 & $-20,99$ & 98,66 & & \\
\hline A17 & 24 & 0.34 & 1.33 & $-20,49$ & 97.85 & & \\
\hline $\mathrm{A} 18$ & 35 & 0,51 & 2,00 & $-19,82$ & 96,76 & & \\
\hline TOT & $\mathrm{NT}=7070$ & 100 & 392,78 & 0 & & 100 & 0 \\
\hline
\end{tabular}

Respecto a la naturaleza de los recursos, se han recodificado según la tipología que muestra la tabla 4. Para los estudiantes, la mitad de los accesos realizados (50,97\%) son de carácter general, a correos y revisión; en torno al 40 \% de los accesos son a los epígrafes de tareas evaluables y recursos disponibles, de forma que el $4,5 \%$ se concentra en foros y chats, el $2,73 \%$ en filiación e identificación, y con menos de $1 \%$ en informes de evaluadores y agrupados y en 
archivos compartidos entre grupos. A diferencia, el profesor8 concentra mayoritariamente su participación en los ítems general (33,62\%), filiación (21,74\%) y tareas evaluables (16,33\%), siendo los mayores diferenciales porcentuales los correspondientes a filiación $(19,01)$ a favor del profesor, mientras que general y recursos disponibles (17,34 y 13,95 respectivamente) es a favor de los estudiantes.

Tabla 4.

Tipología y total de registros curso 2016-17

\begin{tabular}{lcccccr}
\hline & \multicolumn{3}{c}{ Alumnos (NT) } & \multicolumn{2}{c}{ TEACH } & diferencial \\
\hline Tipología Registro internet & Registros & $\%$ & \multicolumn{2}{c}{ Registros } & $\%$ & distror \\
\hline General y sistema=1: T_REG 1 & 3603 & 50,96 & 317 & 33,62 & $-17,34$ \\
\hline Tareas evaluables=2: T_REG 2 & 1437 & 20,32 & 154 & 16,33 & $-4,00$ \\
\hline Filiación e identificación=3: T_REG 3 & 193 & 2,73 & 205 & 21,74 & 19,01 \\
\hline Informes agrupados=4: T_REG 4 & 44 & 0,62 & 75 & 7,95 & 7,33 \\
\hline Recursos disponibles=5: T_REG 5 & 1391 & 19,67 & 54 & 5,73 & $-13,95$ \\
\hline Foros y chats=6: T_REG 6 & 318 & 4,49 & 73 & 7,74 & 3,24 \\
\hline Archivos compartidos=7: T_REG 7 & 29 & 0,41 & 27 & 2,86 & 2,45 \\
\hline Informes evaluadores =8: T_REG 8 & 55 & 0,78 & 38 & 4,03 & 3,25 \\
\hline Total & NT=7070 & 100 & $\mathrm{~N}=943$ & 100 & \\
\hline
\end{tabular}

Los usos digitales por alumno difieren tanto en el cómputo de registros semanales como en la distribución de los mismos según naturaleza de los usos (tabla 5), explicado parcialmente por los roles internos que los estudiantes adquieren en las actividades grupales en las que participan y el uso de otras herramientas al margen del curso (los estudiantes tienen su propio chat).

Tabla 5.

Distribución por alumnos y tipología de recursos, curso 2016-17.

\begin{tabular}{lrrrrrrrrrr}
\hline ALUM & T REG 1 & T REG 2 & T REG 3 & T REG4 & T REG 5 & T REG6 & T REG 7 & T REG 8 & TOT \\
\hline A1 & 51 & 9 & 2 & 0 & 1 & 2 & 1 & 0 & 66 \\
\hline A2_R & 173 & 98 & 16 & 2 & 91 & 24 & 6 & 0 & 410 \\
\hline A3_R & 132 & 83 & 11 & 7 & 84 & 26 & 1 & 0 & 344 \\
\hline A4 & 81 & 60 & 3 & 1 & 27 & 20 & 1 & 0 & 193 \\
\hline A5_R & 375 & 131 & 32 & 21 & 160 & 24 & 5 & 0 & 748 \\
\hline A6_R & 763 & 247 & 37 & 0 & 32 & 0 & 0 & 32 & 1111 \\
\hline A7 & 130 & 59 & 0 & 0 & 0 & 0 & 0 & 12 & 201 \\
\hline A8_R & 585 & 138 & 48 & 2 & 253 & 38 & 4 & 2 & 1070 \\
\hline A9_R & 176 & 117 & 1 & 0 & 82 & 32 & 0 & 0 & 408 \\
\hline A10 R & 243 & 86 & 12 & 2 & 84 & 24 & 4 & 0 & 455 \\
\hline A11_R & 84 & 79 & 1 & 0 & 135 & 24 & 0 & 1 & 324 \\
\hline
\end{tabular}

${ }^{8}$ Obviamente el uso que el profesor hace del campus virtual difiere respecto de sus estudiantes, dado que utiliza cada curso la plantilla del anterior, reduciendo así el uso de ciertas herramientas. 


\begin{tabular}{lrrrrrrrrr}
\hline A12_R & 82 & 76 & 1 & 0 & 31 & 28 & 1 & 0 & 219 \\
\hline A13_R & 124 & 66 & 1 & 0 & 77 & 22 & 0 & 0 & 290 \\
\hline A14_R & 366 & 99 & 13 & 1 & 186 & 28 & 3 & 0 & 696 \\
\hline A15_R & 204 & 78 & 9 & 6 & 142 & 22 & 0 & 0 & 461 \\
\hline A16 & 11 & 2 & 0 & 0 & 2 & 0 & 0 & 0 & 15 \\
\hline A17 & 18 & 4 & 1 & 0 & 1 & 0 & 0 & 0 & 24 \\
\hline A18 & 5 & 5 & 5 & 2 & 3 & 4 & 3 & 8 & 35 \\
\hline TOT & 3603 & 1437 & 193 & 44 & 1391 & 318 & 29 & 55 & 7070 \\
\hline
\end{tabular}

\section{Resultados de las Calificaciones}

Los epígrafes que se evalúan en la aplicación de la metodología ABPD son: la Programación Didáctica (PD) y la Unidad didáctica (UD), con un $25 \%$ respectivamente y el portafolio de actividades y recursos multiformato, con el 50\% restante (PR: póster, pruebas de evaluación, mapa conceptual, ficha evaluación simulador, juego, video, micro investigación, trabajo desde el anuncio, técnicas de fijación, actividad fuera del aula, participación en el blog, etc.). El binomio programación-unidad didáctica que los estudiantes elaboran y defienden públicamente (la programación en grupo y la unidad individualmente), tiene una preevaluación por parte de otro grupo que orienta, guía y prepara para el trabajo en el aula que desarrollarán en el practicum posterior. Por ello, los estudiantes durante el desarrollo del ABPD deben prestar especial atención a i) los objetivos que se fijan en la estrategia docente que programan, ii) las competencias y valores que señalan para su desarrollo y iii) los recursos docentes evaluados y aplicables en la unidad docente.

La fluctuación en notas para el total de estudiantes entre los diversos componentes (PD, UD y PR) y la nota final (NF) es reducida (tabla 6). En media, los estudiantes del trienio objeto del estudio registran una calificación de 8,539 en PD, 8,17 en UD y 8,05 en PR, siendo la calificación media ponderada de 8,32 (desviaciones respectivas 0,439; 0,491; 0,288 y 0,579). Paralelamente, para los estudiantes del curso 2016-17, la calificación en PD es de 8,39, 8,04 en UD y 7,87 en PR con una calificación media ponderada de 8,17.

En el desarrollo de la metodología ABPD los estudiantes participan en diversos grupos de trabajo, seleccionados con diversos criterios (por conocimiento y empatía o por observación previa de su complementariedad competencial), siendo relevante la supervisión del proceso de aprendizaje desde la propia asignación de los grupos de trabajo, hecho que puede hacer que el aprendizaje sea más motivador y cognitivamente enriquecedor (Calzadilla, 2002). Esta asignación por grupos, determina a su vez ciertas dependencias entre los componentes evaluados de la calificación final, que se reflejan en las correlaciones, dado que, si bien las PD se realizan en los grupos prefijados, y de ellas se retroalimentan las UD realizadas individualizadas, son complementadas con los $\mathrm{PR}$, algunos realizados individualmente y otros colectivamente. Así, para el total de estudiantes del trienio, la correlación (Coef. Pearson) entre la calificación en PR y NF es 0,543; y de la PD y la NF es 0,950 y de la UD y la NF es 0,739, mientras que la correlación entre los PR y la UD es 0,109, de PR con la PD 0,423 y de la UD con la PD del 0,574 . 
Tabla 6.

Medias calificaciones y correlaciones. Trienio cursos 2014-15 a 16-17.

\begin{tabular}{llcccc}
\hline & & $\begin{array}{l}\text { Portafolio } \\
\text { PR }\end{array}$ & $\begin{array}{l}\text { Unidad } \\
\text { Didáctica UD }\end{array}$ & $\begin{array}{l}\text { Programación } \\
\text { Docente PD }\end{array}$ & $\begin{array}{l}\text { Nota Final } \\
\text { NT }\end{array}$ \\
\hline Media & Trienio & 8,05 & 8,17 & 8,53 & 8,32 \\
\cline { 2 - 6 } & Curso 2016-17 & 7,87 & 8,04 & 8,39 & 8,17 \\
\hline $\begin{array}{l}\text { Coef. } \\
\text { Correlación }\end{array}$ & PR & & $\mathbf{0 , 1 0 9}$ & $\mathbf{0 , 4 2 3}$ & $\mathbf{0 , 9 5 0}$ \\
\cline { 2 - 6 } $\begin{array}{l}\text { Pearson } \\
\text { Trienio }\end{array}$ & UD & & & $\mathbf{0 , 5 7 4}$ & $\mathbf{0 , 7 3 9}$ \\
\cline { 2 - 6 } & PD & & & & $\mathbf{0 , 5 4 3}$ \\
\hline
\end{tabular}

\section{Resultados de la valoración de la estrategia ABPD}

La tabla 7 muestra los resultados del cuestionario de valoración que los estudiantes realizan de la implementación de la estrategia ABPD, siendo el coeficiente de correlación Pearson de 0,7574 entre la matriz de datos para todos los estudiantes, 0,7678 para aquellos que no autorizan hacer seguimiento (SIN_R), y de 0,9726 para aquellos que sí lo autorizan (CON_R). En general, las valoraciones que realizan del ABPD son más que notables, sobre escala Likert entre 1 y 5 , con medias de 4,26 para el total de estudiantes del trienio analizado, 4,19 para quienes no participan en el seguimiento ( $S I N \_R$ ), y 4,28 para quienes sí (CON_R). Particularizando para los estudiantes del curso 2016-17, siguen el mismo patrón con ligeras diferencias, siendo 4,17 para el total, 4,04 para los que no participan en el seguimiento y 4,23 para los que sí participan.

La valoración positiva de la estrategia $\mathrm{ABPD}^{9}$ está en acercar a los estudiantes a la profesión docente desde diversos frentes: así, señalizan tener una visión más integrada de la profesión docente con 4,21 y 4,15 (respectivamente para los que sí autorizan el SEG y los que no), analizar situaciones de práctica profesional con 4,10 y 4,11 respectivamente), desarrollar competencias necesarias en la práctica profesional con 4,45 y 4,34 (respectivamente), hecho que se acompaña con elevadas calificaciones del curso, si bien opinan que es a costa de un mayor y más continuado esfuerzo, alejado del trabajo puntual más tradicional en otras etapas formativas, que exige mayor actividad, responsabilidad en la autoría y calidad de los recursos y materiales que elaboran. Además, la valoración general del ABPD llega a 4,21 entre quienes participan en el SEG, frente a 4,02 para los que no, consideran que ha mejora el proceso de enseñanza-aprendizaje (4,09 y 3.97 respectivamente), el sistema de evaluación ha sido apropiado (4,41 y 4.38 respectivamente) y las orientaciones del profesor han sido adecuadas (4,29 y 4,19 respectivamente).

Ciertos items del cuestionario ( del I3 al l13, del epígrafe 3) remiten a la autopercepción en el desarrollo de ciertas competencias que el estudiante ejercita, desde buscar información de forma autónoma (4,32 y 4,21 respectivamente para los que sí autorizan el SEG y los que no), desarrollar competencias de comunicación escrita y oral (4, 45 y 4, 34 respectivamente), mostrar actitud participativa en el proceso de aprendizaje (4,43 y 4,30 respectivamente) o aprender a trabajar en equipo (4,52y 4,43 respectivamente), y consideran que la estrategia ABPD favorece desarrollar competencias necesarias en la práctica profesional (4,50 y 4,45 respectivamente).

\footnotetext{
${ }^{9}$ Al no existir grupo de control, impide estimar la causa/efecto, más allá la descripción y valoración que profesorado y alumnos realizan de su implementación.
} 
Tabla 7.

Valoración de la estrategia ABPD, acumulado. Trienio y curso 2016-17.

\begin{tabular}{|c|c|c|c|c|}
\hline & item & \multicolumn{3}{|c|}{$\begin{array}{r}\text { Media Trienio } \\
2014-15 \text { a } 16-17 \\
\end{array}$} \\
\hline A_VALORACION ESTRATEGIA ABPD & & SIN_R & CON_R & TOT \\
\hline 1. Valoración general del ABPD & I1 & 4,02 & 4,21 & 4,18 \\
\hline 2. El proyecto mejora el proceso de enseñanza-aprendizaje & I2 & 3.97 & 4,09 & 4,07 \\
\hline \multicolumn{5}{|l|}{ 3. En qué medida te ha ayudado esta metodología para: } \\
\hline \multirow{6}{*}{$\begin{array}{r}\text { 3.1Tener una visión más integrada de la profesión docente } \\
3.2 \text { Mejorar el interés y la motivación } \\
\text { 3.3 Analizar situaciones de práctica profesional } \\
\text { 3.4 Buscar información de forma autónoma } \\
\text { 3.5 Tomar decisiones en una situación real } \\
\text { 3.6 Solucionar problemas }\end{array}$} & I3 & 4.15 & 4.21 & 4,20 \\
\hline & I4 & 4,02 & 4,22 & 4,18 \\
\hline & $I 5$ & 4.11 & 4.10 & 4,10 \\
\hline & I6 & 4.21 & 4.32 & 4,30 \\
\hline & $I 7$ & 4.07 & 4.05 & 4,05 \\
\hline & I8 & 4.06 & 4.08 & 4,08 \\
\hline \multirow{2}{*}{$\begin{array}{r}3.7 \text { Desarrollar competencias de comunicación escrita y oral } \\
\text { 3.8 Desarrollar autonomía para aprender }\end{array}$} & 19 & 4.34 & 4.45 & 4,43 \\
\hline & $I 10$ & 4.15 & 4.27 & 4,25 \\
\hline \multirow{2}{*}{$\begin{array}{r}\text { 3.9 Mostrar actitud participativa en el proceso de aprendizaje } \\
3.10 \text { Aprender a trabajar en equipo }\end{array}$} & $I 11$ & 4.30 & 4.43 & 4,41 \\
\hline & $I 12$ & 4.43 & 4.52 & 4,50 \\
\hline 3.11 Desarrollar competencias necesarias en la práctica profesional & $I 13$ & 4.45 & 4.50 & 4,49 \\
\hline 4. El sistema de evaluación ha sido el apropiado & I14 & 4.38 & 4.41 & 4,40 \\
\hline 5. Las orientaciones del profesor han sido adecuadas & I15 & 4.17 & 4.29 & 4,27 \\
\hline \multirow[t]{2}{*}{ Media } & item & 4,19 & 4,28 & 4,26 \\
\hline & & \multicolumn{3}{|c|}{ Media Curso 2016-17 } \\
\hline A_VALORACION ESTRATEGIA ABPD & & SIN_R & CON_R & TOT \\
\hline 1. Valoración general del ABPD & I1 & 3,49 & 4,18 & 3,95 \\
\hline 2. El proyecto mejora el proceso de enseñanza-aprendizaje & $\mathbf{I 2}$ & 3,69 & 4,07 & 3,94 \\
\hline \multicolumn{5}{|l|}{ 3. En qué medida te ha ayudado esta metodología para: } \\
\hline \multirow{6}{*}{$\begin{array}{r}\text { 3.1Tener una visión más integrada de la profesión docente } \\
3.2 \text { Mejorar el interés y la motivación } \\
\text { 3.3 Analizar situaciones de práctica profesional } \\
\text { 3.4 Buscar información de forma autónoma } \\
\text { 3.5 Tomar decisiones en una situación real } \\
\text { 3.6 Solucionar problemas }\end{array}$} & I3 & 3,97 & 4.21 & 4,13 \\
\hline & I4 & 3,38 & 4,20 & 3,93 \\
\hline & $I 5$ & 4,04 & 4.14 & 4,11 \\
\hline & I6 & 4,22 & 4.25 & 4,24 \\
\hline & I7 & 4,34 & 4.12 & 4,19 \\
\hline & I8 & 3,92 & 4.09 & 4,03 \\
\hline \multirow{2}{*}{$\begin{array}{r}3.7 \text { Desarrollar competencias de comunicación escrita y oral } \\
3.8 \text { Desarrollar autonomía para aprender }\end{array}$} & 19 & 4,29 & 4.35 & 4,33 \\
\hline & $I 10$ & 3,89 & 4.21 & 4,10 \\
\hline \multirow{2}{*}{$\begin{array}{r}\text { 3.9 Mostrar actitud participativa en el proceso de aprendizaje } \\
3.10 \text { Aprender a trabajar en equipo }\end{array}$} & $I 11$ & 4,23 & 4.37 & 4,32 \\
\hline & $I 12$ & 4,47 & 4.41 & 4,43 \\
\hline 3.11 Desarrollar competencias necesarias en la práctica profesional & $I 13$ & 4,21 & 4.36 & 4,31 \\
\hline 4. El sistema de evaluación ha sido el apropiado & I14 & 4,34 & 4.29 & 4,31 \\
\hline 5. Las orientaciones del profesor han sido adecuadas & I15 & 4,08 & 4.21 & 4,17 \\
\hline Media & & 4,04 & 4,23 & 4,17 \\
\hline
\end{tabular}

En cuanto a la valoración del programa de seguimiento, tabla 8, para el trienio 2014-15 a 16-1 los estudiantes que autorizan el SEG lo valoran con 4,03 (4,23 los del curso 2016-17), consideran que el sistema de evaluación ha sido el adecuado, con medias 4,42 y 4,37 respectivamente, facilita el trabajo en grupo (4,12 y 4,15 respectivamente) y asumir responsabilidades en las aportaciones (4,15 y 4,24 respectivamente). El item referido a si todos los componentes de mi grupo han estado igual de implicados, como medida indirecta del reparto de cargas, trabajo y responsabilidades asumidas en los diversos grupos de trabajo, presenta media de 3,72 y 3,82 respectivamente. 
Tabla 8.

Valoración procedimiento de seguimiento, acumulado Trienio y curso 2016-17.

\begin{tabular}{|c|c|c|c|}
\hline $\begin{array}{l}\text { B_VALORACION SEGUIMIENTO APRENDIZAJE SEG } \\
\text { (alumnos CON_R) }\end{array}$ & item & $\begin{array}{l}\text { Media Trienio } \\
2014-15 \text { a 16-17 }\end{array}$ & $\begin{array}{r}\text { Curso } \\
2016-17\end{array}$ \\
\hline B1. Valoración general del programa de seguimiento & S1 & 4,03 & 4,23 \\
\hline $\begin{array}{l}\text { B2. El seguimiento mejora el proceso de enseñanza- } \\
\text { aprendizaje }\end{array}$ & S2 & 4,01 & 4,05 \\
\hline B3. El seguimiento facilita el trabajo del grupo & S3 & 4,12 & 4,15 \\
\hline $\begin{array}{l}\text { B4. El seguimiento facilita la evaluación de la } \\
\text { participación individual en el grupo. }\end{array}$ & S4 & 4.35 & 4.26 \\
\hline $\begin{array}{l}\text { B5. Todos los componentes de mi grupo han estado } \\
\text { igual de implicados }\end{array}$ & S5 & 3,72 & 3,82 \\
\hline $\begin{array}{l}\text { B.6 El seguimiento facilita asumir responsabilidades en } \\
\text { las aportaciones }\end{array}$ & S6 & 4.15 & 4.24 \\
\hline B7. El sistema de evaluación ha sido el apropiado & S7 & 4.42 & 4.37 \\
\hline B8. Las orientaciones del profesor han sido adecuadas & $\mathbf{S 8}$ & 4.17 & 4.09 \\
\hline Media & & 4,12 & 4,15 \\
\hline
\end{tabular}

\section{Discusión y conclusiones}

Disponer en intervalos temporales tempranos de la información de los registros de usos de los estudiantes, enlazada con las calificaciones y las valoraciones sobre su adaptación a los diversos métodos de aprendizaje, permitirá definir estrategias de aprendizaje ajustadas a la tipología de los estudiantes, incidir en mejorar su rendimiento y compromiso con el programa educativo, hecho que si bien ahora parece impensable, será posible en un futuro a corto plazo, en un paso más de la educación en la era digital. No todos los estudiantes interactúan igual con los recursos docentes, ni tienen el mismo patrón de usos digitales, tampoco han desarrollado igual el corpus competencial (hecho que depende no sólo de la formación recibida, sino también de su entorno social, familiar y vivencial, por experiencias previas), por lo que este tipo de investigaciones, que incorporan la explotación de datos de los registros de usos en las plataformas digitales del curso, empiezan a ser imprescindibles para desarrollar estrategias de aprendizaje adaptables a los perfiles de los estudiantes detectados en etapas tempranas.

El objetivo del trabajo, es integrar elementos derivados del LA con la explotación de la información de los registros de las huellas digitales con las calificaciones de los estudiantes en los distintos componentes de la evaluación final y las valoraciones que los estudiantes realizan sobre el seguimiento autorizado y la implementación de la estrategia metodológica integradora ABPD. Los estudiantes, futuros docentes, participan activamente en la estrategia, siendo responsables de su propio aprendizaje, participando en un programa que reproduce las fases del trabajo docente y que valoran secuencialmente. Cabe destacar como conclusiones más directas, una alta valoración de la estrategia ABPD y del proceso de seguimiento, como instrumentos favorecedores de la adquisición de ciertas competencias. En concreto, se observa un alto diferencial e índices de dispersión en el patrón de usos de los estudiantes en las plataformas digitales, con medias que oscilan del 10\% a 0,05\% del total, y que determinan fuertes brechas en el uso digital. Estos patrones implican una media de 
registros, por estudiante y semana, seis veces superior para aquellos que siguen el plan de seguimiento respecto de aquellos que lo declinan.

De los accesos que realizan los estudiantes a las plataformas digitales, el $50 \%$ son de carácter general para supervisar cambios, correos, revisiones y el $40 \%$ a tareas evaluables y recursos disponibles, mostrando un perfil muy tradicional en los usos. Comparando el patrón de uso del profesor con respecto a los estudiantes, los mayores diferenciales porcentuales se encuentran en los usos de filiación $(19,01)$ a favor del profesor, y en los aspectos generales y recursos disponibles $(3,39)$ a favor de los estudiantes.

Las valoraciones que realizan los estudiantes del ABPD son mayores entre quienes autorizan el seguimiento (4,28 frente a 4,19 de quienes no autorizan), y los ítems más significativos son tener una visión más integrada de la profesión docente (4,21 frente a 4,15), analizar situaciones de práctica profesional $(4,10$ y 4,11) y desarrollar competencias necesarias en la práctica profesional $(4,45$ y 4,34$)$. Además, consideran que ha mejora el proceso de enseñanza-aprendizaje (4,09 y 3.97), el sistema de evaluación ha sido apropiado (4,41 y 4.38) y también las orientaciones del profesor (4,29 y 4,19).

En la sección que muestra la autopercepción de los estudiantes en el desarrollo de competencias, valoran buscar información de forma autónoma (4,32 y 4,21 respectivamente), desarrollar competencias de comunicación escrita y oral (4,45 y 4,34), mostrar actitud participativa en el proceso de aprendizaje $(4,43$ y 4,30$)$ o aprender a trabajar en equipo $(4,52$ y 4,43).

Respecto a la valoración del programa de seguimiento, los estudiantes consideran que el sistema de evaluación ha sido el adecuado (4,42 y 4,37 respectivamente), facilitando el seguimiento el trabajo en grupo $(4,12$ y 4,15$)$ y asumir responsabilidades en las aportaciones $(4,15$ y 4,24$)$.

Estas consideraciones muestran la necesidad de incidir en investigaciones de este ámbito ya que, con limitaciones, suponen una aportación innovadora al campo de la implementación de metodologías integrales y activas diseñadas específicamente para la formación de futuros docentes, observadas y analizadas con instrumentos cuantitativos diversos, recuperando la información o huella que su tránsito deja por las aulas virtuales, combinado con las evaluación del programa formativo y de las competencias desarrolladas. Algunas de estas conclusiones orientan hacia futuras líneas de investigación, como también apuntan Ferguson, Brasher, Clow y otros (2016), referidas a conocer los hábitos de los estudiantes durante el proceso de aprendizaje, desequilibrios en el desarrollo de competencias y habilidades, interacción en el trabajo en equipo y colaborativo, etc.

Recuperando el inspirador trabajo de Long y Siemens (2011), cabe concluir que el LA está permitiendo a las Instituciones Educativas ver, entre la niebla de la incertidumbre, cómo asignar eficientemente los recursos, desarrollar ventajas competitivas y, más aún, mejorar la calidad y el valor de la experiencia de aprendizaje, tanto para el docente como los estudiantes, convirtiéndose, en un breve espacio de tiempo, en una realidad ofrecer una enseñanza más orientada a la tipología del estudiante. Este trabajo contribuye a tener una radiografía inicial del comportamiento de los estudiantes en su interacción con las herramientas digitales del curso y las calificaciones y sus valoraciones, en la aplicación de una metodología de aprendizaje activa e integradora, ABPD, diseñada para la formación de futuros docentes. 


\section{Referencias}

Bates, A.W. (T) (2015). Teaching in a Digital Age: Guidelines for designing teaching and learning for a digital age. University of British Columbia. https://opentextbc.ca/teachinginadigitalage/

Berden, W. (2012). Project-Based Learning: Differentiating Instruction for the $21^{\text {st }}$ Century. California: Corwin.

Boss, S. y Krauss, J. (2007). Reinventing Project-Based Learning: your field-guide to real-word projects in the Digital Age. Washington-EEUU: ISTE.

Calzadilla, M. E. (2002). Aprendizaje colaborativo y tecnologías de la información y la comunicación. Revista Iberoamericana de Educación, 29(1), 1-10. https://rieoei.org/RIE/article/view/2868

Campbell, J.P. (2007). Utilizing Student Data within the Course Management System to Determine Undergraduate Student Academic Success: An Exploratory Study, PhD, Purdue University.

Cano, R. (2009). Tutoría universitaria y aprendizaje por competencias ¿Cómo lograrlo? Revista Electrónica Interuniversitaria de Formación del Profesorado, 12 (1), 181-204. http://www.redalyc.org/articulo.oa?id=217015332014

Ferrari, A. (2013) A Framework for Developing and Understanding Digital Competence in Europe. Institute for Prospective Technological Studies, (http://publications.jrc.ec.europa.eu/repository/bitstream/JRC83167/lb-na-26035enn.pdf

Leah P. M. and Dawson S., Mining LMS Data to Develop an 'Early Warning System' for Educators: A Proof of Concept, Computers \& Education, vol. 54, no. 2 (2010), pp. 588599.

Ferguson, R. (2012). Learning analytics: drivers, developments and challenges. International Journal of Technology Enhanced Learning, 4(5/6) pp. 304-317.

Ferguson, R. Brasher A. Clow D. y otros (2016). Research Evidence on the Use of Learning Analytics - Implications for Education Policy. R. Vuorikari, J. Castaño Muñoz (Eds.). Joint Research Centre Science for Policy Report; EUR 28294 EN; doi:10.2791/955210.

Harrison, C y Killion, J. (2007). Ten roles for teachers leaders. Educational Leadership, v65 n1 (September) p74-77.

http://www.ascd.org/publications/educational-leadership/septo7/vol65/num01/ten-

roles-for-teacher-leaders.aspx

Herrero, R. (2014). El papel de las TIC en el aula universitaria para la formación en competencias del alumnado. Pixel-Bit. Revista de Medios y Educación, (45), 173-188. https://idus.us.es/xmlui/handle/11441/46215

Iglesia, M. C. (2018). Aprendizaje Basado en un Proyecto Docente: Aprendizaje, creatividad, innovación y nuevos roles en la formación de profesorado en la era digital. Revista Complutense de Educación, 29(4) 2018: 1253-1278.

Iglesia, M. C. (2006). Varios retos y un único destino en la adaptación de los contenidos de la Teoría Económica al nuevo contexto de enseñanza aprendizaje. El campus virtual como herramienta de apoyo docente. Aplicaciones prácticas de la convergencia europea. Actas Jornadas nacionales de intercambio de experiencias piloto de implantación de metodologías ECTS. 
Kaura, P., Singhb, M. y Josanc, G.S. (2015). Classification and prediction based data mining algorithms to predict slow learners in education sector. 3rd International Conference on Recent Trends in Computing (ICRTC-2015)

Long, Ph. y Siemens, G. (2011). "Penetrating the Fog: Analytics in Learning and Education". EDUCAUSE Review, v46 n5 p30-40 Sep-Oct 2011.

Patton, A. (2012). Work that matters. The Teacher's guide to project-based learning. Londres: Paul Hamlyn Foundation.

http://www.innovationunit.org/sites/default/files/teacher's\%2oguide\%20to\%2oprojec t-based\%2olearning.pdf

Prensky, M. (2013). Enseñar a nativos digitales (1a. ed). México: SM Ediciones, 240 pp.

Romero, C., y Ventura, S. (2007). Educational data mining: A survey from 1995 to 2005. Expert Systems with Applications, 33(1), 135-146.

Romero, C., Ventura S., y García, E. (2008). Data mining in course management systems: Moodle case study and tutorial. Computers \& Education, 51(1), pp. 368-384,

Sampson, D. (2017). Teaching and learning analytics to support teacher inquiry. In 2017 IEEE Global Engineering Education Conference (EDUCON 2017), Apr 25, 2017, Athens, Greece.

Siemens, G. y Baker, R. S. J. (2012). Learning analytics and educational data mining: towards communication and collaboration. In Proceedings of the 2nd International Conference on Learning Analytics and Knowledge (LAK '12), Buckingham, Gasevic, and Ferguson (Eds.). ACM, New York, NY, USA, 252-254. DOI=http://dx.doi.org/10.1145/2330601.2330661

Siemens, G., Dawson S., and Lynch G., (2013). Improving the Quality of Productivity of the Higher Education Sector: Policy and Strategy for Systems-Level Deployment of Learning Analytics, SoLAR. http://bit.ly/1KNZgHH

Vela-Pérez, M., Hernández-Estrada, A.,Tirado-Domínguez, G. y otros (2017). Learning Analytics to classify students according to their activity in moodle. EDULEARN17 Proceedings, pp. 1166-1172. Barcelona.

Villalustre, L. y Del Moral M. E. (2014). Digital storytelling: una nueva estrategia para narrar historias y adquirir competencias por parte de los futuros maestros. Revista Complutense de Educacion, 25(1), p. 115-132 (2014). http://revistas.ucm.es/index.php/RCED/article/view/41237

Viñals, A. y Cuenca, J. (2016). El rol del docente en la era digital. Revista Interuniversitaria de Formación del Profesorado, 86 (30.2), 103-114. http://www.redalyc.org/pdf/274/27447325008.pdf 


\section{Anexo}

Ficha metodología integrada ABPD

Experto en contenidos docentes.

Diseñador de nuevos entornos de aprendizaje.

Nuevos roles del

Generador y evaluador de recursos tecnológicos y multiformato.

docente

Orientador y Tutor.

Motivador en el aprendizaje.

Co-aprendiz de su profesión.

Acompañante en el proceso formativo.

\begin{tabular}{|c|c|}
\hline $\begin{array}{l}\text { Propuesta } \\
\text { Integral }\end{array}$ & Elementos: creatividad, digitalidad y aprender la profesión. \\
\hline Uso TIC & $\begin{array}{l}\text { Formación centrada en el estudiante. } \\
\text { Adaptable según características y necesidades. } \\
\text { Flexible. } \\
\text { Superadora de barreras espacio-temporales. } \\
\text { Generadora de contenidos cuasi-inmediatos. } \\
\text { Extensión masiva. } \\
\text { Mayor interacción y comunicación. } \\
\text { Nuevos espacios de aprendizaje. } \\
\text { Aprendizaje cooperativo. } \\
\text { Canales de evaluación. } \\
\text { Gestión y responsabilidad de la autoría. }\end{array}$ \\
\hline Recursos & $\begin{array}{l}\text { Visión Radial. } \\
\text { Multidisciplinar. } \\
\text { Orientado a la síntesis. } \\
\text { Actualizado. } \\
\text { Espacio docente compartido. }\end{array}$ \\
\hline Metodología & $\begin{array}{l}\text { Adaptabilidad. } \\
\text { Aprender haciendo. } \\
\text { Estimulación creativa. } \\
\text { Modelar los usos digitales. } \\
\text { Aspectos sociales. } \\
\text { Dinámismo. } \\
\text { Interactividad inter, intra. }\end{array}$ \\
\hline $\begin{array}{l}\text { Materiales } \\
\text { docentes }\end{array}$ & $\begin{array}{l}\text { Imagen: Mensaje claro e inequívoco. Originalidad/autoría. } \\
\text { Mensaje: Util y eficaz en la transmisión del concepto. } \\
\text { Elaboración tema: Conocimiento técnico e interrelación. } \\
\text { Síntesis: Extraer lo significativo: mapas conceptuales. } \\
\text { Evaluación: Métodos diversos, diversos soportes, usos creativos. }\end{array}$ \\
\hline Estructura & $\begin{array}{l}\text { Programación: trabajo en equipo. } \\
\text { Evaluación por pares: feed back. } \\
\text { Unidad didáctica: individual. } \\
\text { Recursos docentes: objetivos de la estrategia, competencias y valores: individual } \\
\text { y en grupos. }\end{array}$ \\
\hline $\begin{array}{l}\text { Nuevos roles } \\
\text { del docente }\end{array}$ & $\begin{array}{l}\text { Experto en contenidos docentes. } \\
\text { Diseñador de nuevos entornos de aprendizaje. } \\
\text { Generador y evaluador de recursos tecnológicos y multiformato. } \\
\text { Orientador y Tutor. } \\
\text { Motivador en el aprendizaje. } \\
\text { Co-aprendiz de su profesión. } \\
\text { Acompañante en el proceso formativo. }\end{array}$ \\
\hline $\begin{array}{l}\text { Propuesta } \\
\text { Integral }\end{array}$ & Elementos: creatividad, digitalidad y aprender la profesión. \\
\hline Uso TIC & rmación centrada en el estudiante. \\
\hline
\end{tabular}




\begin{tabular}{|c|c|}
\hline & $\begin{array}{l}\text { Adaptable según características y necesidades. } \\
\text { Flexible. } \\
\text { Superadora de barreras espacio-temporales. } \\
\text { Generadora de contenidos cuasi-inmediatos. } \\
\text { Extensión masiva. } \\
\text { Mayor interacción y comunicación. } \\
\text { Nuevos espacios de aprendizaje. } \\
\text { Aprendizaje cooperativo. } \\
\text { Canales de evaluación. } \\
\text { Gestión y responsabilidad de la autoría. }\end{array}$ \\
\hline Recursos & $\begin{array}{l}\text { Visión Radial. } \\
\text { Multidisciplinar. } \\
\text { Orientado a la síntesis. } \\
\text { Actualizado. } \\
\text { Espacio docente compartido. }\end{array}$ \\
\hline Metodología & $\begin{array}{l}\text { Adaptabilidad. } \\
\text { Aprender haciendo. } \\
\text { Estimulación creativa. } \\
\text { Modelar los usos digitales. } \\
\text { Aspectos sociales. } \\
\text { Dinamismo. } \\
\text { Interactividad inter, intra. }\end{array}$ \\
\hline $\begin{array}{l}\text { Materiales } \\
\text { docentes }\end{array}$ & $\begin{array}{l}\text { Imagen: Mensaje claro e inequívoco. Originalidad/autoría. } \\
\text { Mensaje: útil y eficaz en la transmisión del concepto. } \\
\text { Elaboración tema: Conocimiento técnico e interrelación. } \\
\text { Síntesis: Extraer lo significativo: mapas conceptuales. } \\
\text { Evaluación: Métodos diversos, diversos soportes, usos creativos. }\end{array}$ \\
\hline Estructura & $\begin{array}{l}\text { Programación: trabajo en equipo. } \\
\text { Evaluación por pares: feed-back. } \\
\text { Unidad didáctica: individual. } \\
\text { Recursos docentes: objetivos de la estrategia, competencias y valores: } \\
\text { individual y en grupos. }\end{array}$ \\
\hline
\end{tabular}

